\title{
The role of coping strategies in quality of life of adolescents with asthma
}

\author{
Monique O. M. Van De Ven • \\ Rutger C. M. E. Engels · Susan M. Sawyer · \\ Roy Otten · Regina J. J. M. Van Den Eijnden
}

Received: 24 May 2006/ Accepted: 26 October 2006/ Published online: 1 February 2007

(C) Springer Science+Business Media B.V. 2007

\begin{abstract}
This study aimed to determine which coping strategies are associated with better quality of life (QOL) in adolescents with asthma. Furthermore, because coping can mediate the effects of health stressors on QOL, this study also examined the indirect effect of asthma severity on QOL via coping. Between January and May 2003, 553 Dutch adolescents with asthma (aged 12-16 years) completed questionnaires assessing coping strategies, symptom severity, overall QOL and positive QOL domain (positive effects of asthma domain). The relations between symptom severity, coping strategies and QOL were tested with structural equation modelling (SEM). Symptom severity affected overall and positive QOL, both directly and indirectly via coping. The coping strategies restricted lifestyle and
\end{abstract}

M. O. M. Van De Ven $(\varangle)$ · R. C. M. E. Engels ·

R. Otten · R. J. J. M. Van Den Eijnden

Behavioural Science Institute, Radboud University

Nijmegen, P.O. Box 9104, 6500 HE Nijmegen,

The Netherlands

e-mail: M.vandeVen@pwo.ru.nl

\section{S. M. Sawyer}

Centre for Adolescent Health, Royal Children's Hospital, Melbourne, VIC, Australia

S. M. Sawyer

Department of Paediatrics, The University of Melbourne,

Parkville, VIC, Australia

\section{S. M. Sawyer}

Murdoch Childrens Research Institute, Melbourne,

VIC, Australia

R. J. J. M. Van Den Eijnden

IVO, Addiction Research Institute, Rotterdam,

The Netherlands worrying about asthma were associated with poorer overall QOL. The use of the coping strategies restricted lifestyle, positive reappraisal, and information seeking was related to increased scores on the positive QOL domain, whereas hiding asthma was related to lower scores on the positive QOL domain. The findings of this study suggest that coping mediates the effect of symptoms of asthma on QOL among adolescents with asthma. Several suggestions for interventions to improve asthma-specific QOL are discussed.

Keywords Psychological adaptation - Adolescent . Asthma - Quality of life
Abbrevations
QOL Quality of life
ISAAC International Study of Asthma and
$\begin{array}{ll}\text { Allergies in Childhood } \\ \text { SES } & \text { Socio economic status }\end{array}$
ACAAI American College of Allergy,
Asthma and Immunology
AAQOL Adolescent Asthma Quality of Life
Questionnaire
SEM Structural equation modelling
CFI Comparative fit index
RMSEA Root mean square error of approximation

Asthma is the most common chronic disease in childhood and adolescence in Western countries. While adolescence can be a tumultuous period for many youngsters, adolescents with asthma encounter 
additional problems due to the need for regular medication and limitation to sporting activities and social events [1]. More significant effects on peer relationships are reported, including stigmatisation [2], especially when hospitalisation is required [3].

Quality of life (QOL) has been defined as a multidimensional concept, including the physical, psychological and social impact a disease has on the daily life of a patient [4]. Several studies demonstrate reduced QOL in children and adolescents with asthma when compared to their healthy peers [5]. In adults, it has been shown that QOL strongly depends on symptom frequency [6]. In children and adolescents, increased asthma severity is also associated with poorer QOL $[5,7,8]$.

Although severity has a negative impact on QOL of patients with asthma, adequate coping strategies may mediate these negative effects $[9,10]$. In this study, coping is defined as a set of behavioural and cognitive responses designed to manage the stressors of a situation [11]. In adults with asthma, emotional coping (a passive affective reaction to the problem) and avoidant coping (ignoring, denying or avoiding a problem) are associated with poorer QOL $[9,10]$, whereas active coping (taking an active approach to the problem, whether cognitive or behavioural) is associated with better QOL [9]. To our knowledge, no studies have examined the impact of coping on QOL in adolescents with asthma. Because of the specific issues adolescents encounter in comparison to adults, school absence, the role of parents, and concerns about peer relationships, it may not be possible to generalise the findings for adults to adolescents.

The goal of the present study was to determine which coping strategies are associated with a better QOL among adolescents with asthma. It is hoped that identification of these strategies will provide important directions for designing interventions aimed at improving QOL in adolescents with asthma. Coping strategies were found to mediate the relation between symptom severity and QOL in patients with other chronic conditions [12]. We hypothesised that coping strategies could also mediate the effect of asthma severity on QOL in adolescents with asthma. Therefore, we also aimed to examine the indirect relation between asthma severity and QOL via coping. Because girls report more symptoms of asthma [13] and lower QOL [7] than boys do, but seem better adapted to living with asthma [14], we also examined gender differences in coping, severity, and QOL, as well as the relationships between these concepts.

\section{Methods}

Sample and sampling procedure

The present study uses data from a national sample of Dutch adolescents with and without asthma [13, 15] that was collected between January and May 2003. This study was approved by the medical ethics committee (CMO Arnhem-Nijmegen). Fifty-five government-funded secondary schools in four regions of the Netherlands were randomly selected and approached for participation; 33 schools agreed to participate in this study. The main reason for refusal was because of participation in other studies. The majority of secondary schools in the Netherlands are privately managed, but over 99 percent of schools are funded by the government.

All students from the first two years of secondary school were asked to fill out written questionnaires during school hours, under the supervision of a teacher. A total of $9008(89.3 \%)$ students aged $12-16$ years fully completed the questionnaires. Adolescents with asthma were identified using an extended version of the asthma questionnaire of the International Study of Asthma and Allergies in Childhood (ISAAC) [16], translated into Dutch [17]. The ISAAC questionnaires are designed for population-based research and have proven to be valid instruments for measuring the prevalence of atopic diseases [16, 18, 19]. Furthermore, the results of the asthma questionnaire have been compared with a physician's assessment of asthma and demonstrated both sensitivity and specificity [20]. Respondents were selected based on their positive responses to three items: students who reported lifetime asthma and who either reported they had suffered from asthma in the past 12 months or who had used asthma medication in the past 12 months were categorized as currently having asthma. This resulted in a final sample of 553 adolescents with asthma. The respondents had suffered from asthma for a mean period of 6.74 years (SD 4.23).

The mean age of the respondents was 13.33 years (SD 0.83), of whom 286 (52\%) were girls. Most students were of Dutch origin (84.6\%) and lived in twoparent families $(82.2 \%)$. The distribution of the education level of the father implied that all socio-economic status (SES) classes are represented in the sample. However, there is a slight over representation of lower SES classes compared to the general Dutch population, consistent with a higher prevalence of asthma in lower SES classes. 
Measures

\section{Symptom severity}

Severity of asthma symptoms was assessed among all adolescents using a Dutch translation of the student questionnaire of the American College of Allergy, Asthma and Immunology (ACAAI) [21]. Respondents were asked to indicate on a 3-point scale (never, sometimes, a lot) how often they suffered from seven symptoms (e.g., "I wake up at night because I have trouble breathing"). Responses were averaged to form a severity score (Cronbachs alpha $(\alpha)=.72$ ); higher scores indicate more severe asthma.

\section{Quality of life}

QOL was only measured among the adolescents with asthma, using a Dutch translation of the Adolescent Asthma Quality of Life Questionnaire (AAQOL) [22], the only asthma-specific QOL instrument designed specifically for this age group, which covers domains of particular relevance for adolescents. This instrument has been validated among Australian and European adolescents [8], and shows good construct validity and high test-retest reliability [22]. The AAQOL questionnaire consists of six dimensions: the symptoms domain (six items, e.g., "How bothered have you been by coughing", $\alpha=.89$ ), the medication domain (five items that tap the concerns adolescents have about their medication, e.g., "How bothered did you feel about having to carry your inhaler with you", $\alpha=.74$ ), the physical activities domain (six items, e.g., "How often have you been restricted in sports, hobbies or other recreational activities because of your asthma", $\alpha=.84$ ), the emotions domain (five items, e.g., "How often did you feel frustrated because of your asthma", $\alpha=.91$ ), the social interaction domain (five items measuring the impact asthma has on the social life of adolescents, e.g., "How often have you been annoyed by your asthma when going to a party", $\alpha=.73$ ), and the positive effects domain (five items, $\alpha=.75$, e.g., "How often has your asthma brought you closer as a family"). All items were measured on a 7-point Likert scale; mean scores were calculated for each domain. However, because of the possible overlap between the symptoms domain and the severity scale, the symptoms domain was not included in the analyses. As recommended by the authors, the first domains are added together to create one scale called overall $Q O L$ $(\alpha=.93)$. The positive effects domain could not be included in the overall score because it cannot be meaningfully added to the total score [22]. Higher scores represent a better QOL on that domain.

\section{Coping strategies}

Coping strategies were assessed in adolescents with asthma, using the Asthma-Specific Coping Scale [23], a modification of the trait form of the Asthma Coping Scale [24]. The psychometric characteristics of this questionnaire have been tested in a validation study among a large general asthma sample of 3,459 adults and in two smaller intervention samples of adults [23]. The results of this validation study supported construct, concurrent and discriminant validity, and Cronbachs alpha's ranged from .63 to .84 . The scale comprises six subscales: restricted lifestyle (four items, e.g., "I avoid exertion", $\alpha=.69$ ), hiding asthma (four items, e.g., "I avoid talking about my asthma", $\alpha=.71$ ), positive reappraisal (four items, e.g., "I try to learn something positive about my falling ill and related experiences", $\alpha=.60$ ), information seeking (four items, e.g., "I try to find out more about my asthma", $\alpha=.72$ ), ignoring asthma (four items, e.g., "I avoid thinking about my asthma", $\alpha=.71$ ) and worrying about asthma (three items, e.g., "I am afraid that my asthma will get worse", $\alpha=.63$ ). All items are measured on a 4-point Likert scale (hardly ever to almost always). Responses were averaged to yield a score for each domain; higher scores indicated more use of that coping strategy.

\section{Demographic information}

Gender, age and SES (assessed by the highest education level of the father) were included in the analyses as possible confounders.

\section{Data analysis}

The analyses were conducted in four steps. First, gender differences in severity, coping strategies and QOL were examined using $t$-tests. Second, Pearson correlation coefficients between severity, coping and the QOL domains were calculated. Third, the relations between severity, coping and QOL were tested with structural equation modelling (SEM) using the program Amos 5 [25]. SEM makes it possible to combine confirmatory factor analysis with multiple regressions. By using underlying, unobserved (latent) variables with multiple indicators, measurement error can be reduced. Furthermore, it is possible to specify and estimate hypothesized causal pathway models with multiple 
mediating variables and multiple outcome variables. Therefore, the SEM approach is useful in determining the relations between severity, coping with asthma and QOL.

Although the variables in this study have an ordinal character, because of the Likert scales used, they are treated as interval scales. Likert [26] proposed to formulate scales with equal appearing intervals in the format we used for the items in this study. Togerson [27] developed scaling methods to support the idea that Likert scales can be treated as interval scales, at method which is now widely accepted.

Maximum likelihood estimation was used to test the model illustrated in Figure 1. This model has 66 degrees of freedom, and our sample size of 553 is sufficient to achieve power of 0.80 [28]. All variables in the model had a normal distribution with skewness and kurtosis between -1 and +1 , except for the coping subscale hiding asthma, which had a skewness of 1.09. The latent variable overall $Q O L$ was measured by four observed indicators: the scores on the first four subscales of the AAQOL questionnaire. The latent variable severity had three parcels as indicators. Parcels are subsets of scale items. Items with corresponding factor loadings were divided over three subsets, leading to three parcels for severity [29]. The other constructs in the model-the coping strategies and positive QOL-correspond to the observed mean scores for these variables. As shown in Figure 1, all direct paths between severity and coping strategies and between severity and QOL were estimated. Furthermore, we tested indirect paths between severity and QOL via coping. The effects of age, gender and SES were modelled by direct paths of these possible confounders to severity, the six coping strategies, overall QOL and positive QOL. However, for clarity, these paths are not shown in Figure. 1

The goodness of fit of the model was evaluated with several fit indices. The chi-square value with degrees of freedom and the $P$-value of the model are calculated. However, the chi-square is extremely sensitive to sample size: in a large sample, it is very likely that the chi-square statistics indicates that the model needs to be rejected, even if it describes the data quite well. Therefore, other fit measures (independent of $\mathrm{N}$ ) were included to evaluate the fit. This study evaluated a chisquare test based index, a comparative-fit index and an absolute-fit index, respectively the chi-square to degrees of freedom ratio $\left(\chi^{2} / \mathrm{df}\right)$, the comparative fit index (CFI) and the root mean square error of approximation (RMSEA). A model that fits the data well should have good results for many different fit

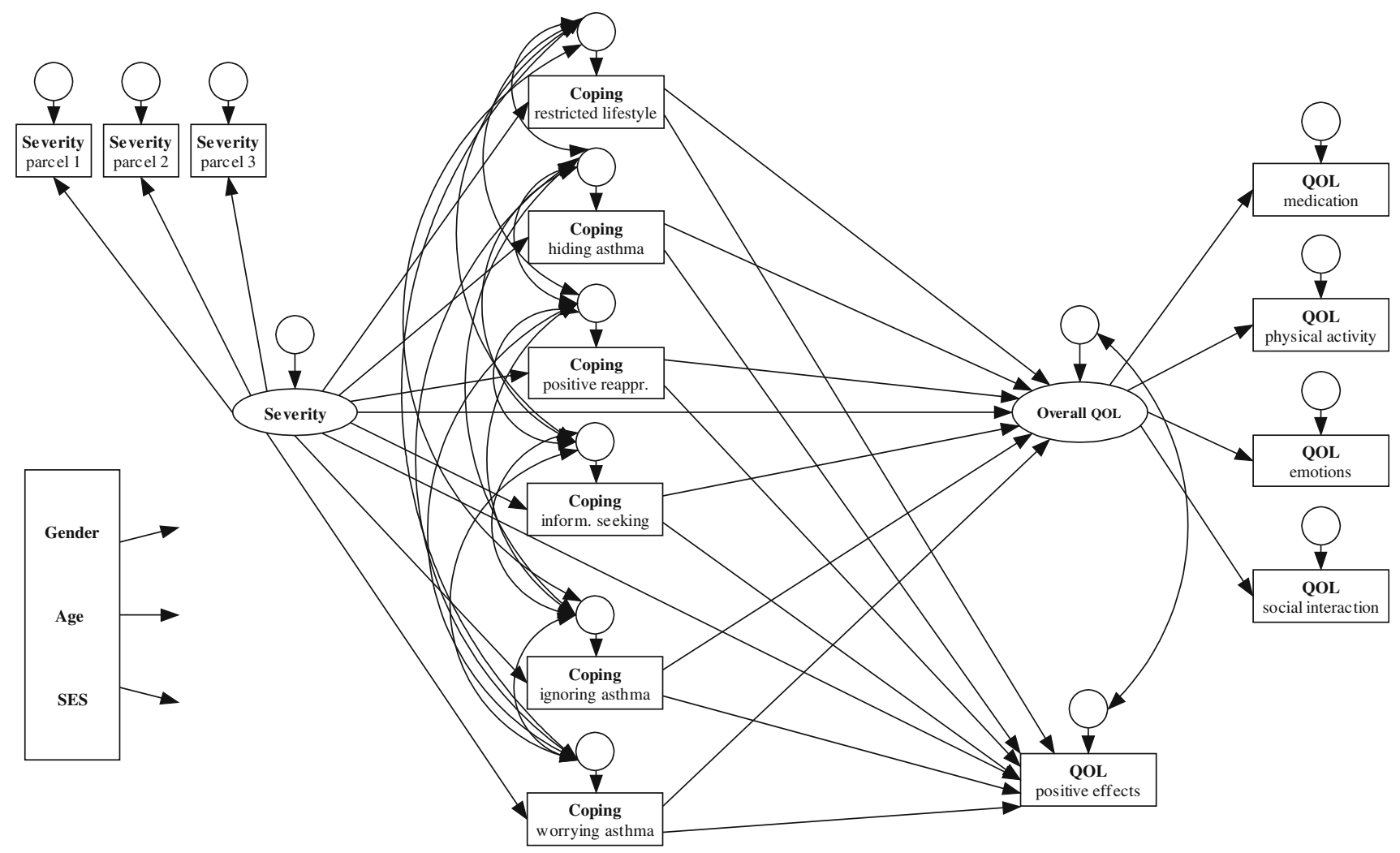

Fig. 1 Conceptual model 
indices. The $\chi^{2} / \mathrm{df}$ ratio should be less than three; for CFI, values greater than 0.90 suggest an acceptable fit between the model and the data, values greater than 0.95 indicate a good fit [30]. For RMSEA, values below 0.08 indicate an acceptable fit; values below 0.05 indicate a good fit [30]. In the last step of the analyses, we tested gender differences by multigroup analyses. A nested model with two separate groups (boys and girls) was tested. Differences in structural parameters were tested by chi-square difference testing.

\section{Results}

\section{Descriptive statistics}

Table 1 summarizes descriptive statistics of the main study variables for the total group, as well as comparisons between boys and girls. Gender differences were found for severity, coping and QOL. Girls reported higher asthma symptom severity than boys. The most commonly reported coping strategies in both boys and girls were hiding asthma and positive reappraisal. Girls had higher scores for three coping strategies: restricted lifestyle, information seeking and worrying about asth$m a$. With regard to QOL, gender differences were found for the first four QOL subscales, where boys reported higher QOL than girls. Boys and girls did not differ in regard to the positive effects domain.

Correlations between the variables of the model are outlined in Table 2. All coping scales were positively interrelated except for two combinations (hiding asthma with positive reappraisal, and hiding asthma with information seeking). The first four QOL subscales were strongly and positively interrelated and the positive QOL domain showed moderate negative correlations with all other QOL subscales, supporting the integration of the first four subscales (and not the positive effects domain) into one overall QOL score.

\section{Structural equation model}

To facilitate presentation, the standardised factor loadings of the latent variables as well as the interrelations of the coping strategies and the interrelation between positive QOL and overall QOL are presented in Table 3, and not in the graphic representation of the model in Figure 2. The standardised factor loadings for severity vary between .60 and .76, thus indicating that the latent variable is very well represented by these indicators. For overall $Q O L$, the standardised factor loadings vary between .69 and .91, indicating that a higher-order factor existed, and that the latent variable overall $Q O L$ is very well represented by the indicators.

Figure 2 shows the significant paths of the model with standardised regression weights. The fit indices showed that the model fitted the data well $\left(\chi^{2} / \mathrm{df}\right.$ ratio below 3; CFI was above 0.95, RMSEA was below 0.08). Nineteen percent of the variance in the positive QOL domain and $56 \%$ of the variance in overall QOL was explained by the model. In regard to the relationships between the possible confounders and severity, coping and QOL, only the relation between gender and severity was significant, with severity being higher among girls. Gender was not related to coping or QOL in the total model. Furthermore, age and SES did not relate to any of the variables in the model.

\section{Coping and $Q O L$}

The overall QOL score was associated with the coping strategies restricted lifestyle and worrying: the more

Table 1 Means, ranges and standard deviations for the total group, and separately for boys and girls

\begin{tabular}{|c|c|c|c|c|c|}
\hline & Total group & Boys & Girls & $t$-value & $P$ \\
\hline $\begin{array}{l}\text { Symptom severity } \\
\text { Coping }\end{array}$ & $1.77(1.00-3.00 ; 0.38)$ & $1.69(1.00-3.00 ; 0.37)$ & $1.84(1.00-3.00 ; 0.39)$ & -4.79 & $<0.001$ \\
\hline Restricted lifestyle & $1.80(1.00-4.00 ; 0.68)$ & $1.70(1.00-4.00 ; 0.67)$ & $1.89(1.00-4.00 ; 0.67)$ & -3.31 & $<0.001$ \\
\hline Hiding asthma & $1.91(1.00-4.00 ; 0.69)$ & $1.87(1.00-4.00 ; 0.65)$ & $1.95(1.00-4.00 ; 0.71)$ & -1.31 & $\mathrm{~ns}$ \\
\hline Positive reappraisal & $2.19(1.00-4.00 ; 0.70)$ & $2.15(1.00-4.00 ; 0.74)$ & $2.22(1.00-4.00 ; 0.67)$ & -1.03 & $\mathrm{~ns}$ \\
\hline Information seeking & $1.72(1.00-4.00 ; 0.66)$ & $1.66(1.00-4.00 ; 0.65)$ & $1.78(1.00-4.00 ; 0.67)$ & -2.12 & $<0.05$ \\
\hline Ignoring asthma & $1.87(1.00-4.00 ; 0.75)$ & $1.84(1.00-4.00 ; 0.74)$ & $1.90(1.00-4.00 ; 0.77)$ & -0.92 & ns \\
\hline $\begin{array}{l}\text { Worrying asthma } \\
\text { Quality of Life }\end{array}$ & $1.64(1.00-4.00 ; 0.67)$ & $1.55(1.00-4.00 ; 0.63)$ & $1.73(1.00-4.00 ; 0.71)$ & -3.04 & $<0.01$ \\
\hline Medication & $5.41(1.60-7.00 ; 1.18)$ & $5.53(1.60-7.00 ; 1.19)$ & $5.31(2.00-7.00 ; 1.16)$ & 2.09 & $<0.05$ \\
\hline Physical activities & $4.62(1.00-7.00 ; 1.43)$ & $4.94(1.60-7.00 ; 1.31)$ & $4.32(1.00-7.00 ; 1.47)$ & 5.14 & $<0.001$ \\
\hline Emotions & $5.31(1.20-7.00 ; 1.43)$ & $5.56(1.40-7.00 ; 1.33)$ & $5.08(1.20-7.00 ; 1.48)$ & 3.99 & $<0.001$ \\
\hline Social interaction & $5.51(1.60-7.00 ; 1.16)$ & $5.69(2.60-7.00 ; 1.03)$ & $5.34(1.60-7.00 ; 1.25)$ & 3.58 & $<0.001$ \\
\hline Positive effects & $3.54(1.00-7.00 ; 1.39)$ & $3.46(1.00-7.00 ; 1.41)$ & $3.61(1.00-6.60 ; 1.37)$ & -1.31 & ns \\
\hline
\end{tabular}

Note: Data are expressed in means; values in parentheses represent ranges and standard deviations 
Table 2 Pearson correlations between model variables

\begin{tabular}{|c|c|c|c|c|c|c|c|c|c|c|c|c|}
\hline & \multirow{2}{*}{$\begin{array}{l}\text { Severity } \\
1\end{array}$} & \multicolumn{6}{|l|}{ Coping } & \multicolumn{5}{|l|}{ QOL } \\
\hline & & 2 & 3 & 4 & 5 & 6 & 7 & 8 & 9 & 10 & 11 & 12 \\
\hline \multicolumn{13}{|l|}{ 1. Severity } \\
\hline \multicolumn{13}{|l|}{ Coping } \\
\hline 2. Restricted lifestyle & $.32 * * *$ & & & & & & & & & & & \\
\hline 3. Hiding asthma & $.12 * *$ & $.16^{* * *}$ & & & & & & & & & & \\
\hline 4. Positive reappraisal & $.11 * *$ & $.36^{* * *}$ & -.01 & & & & & & & & & \\
\hline 5. Information seeking & $.22 * * *$ & $.42 * * *$ & .03 & $.53 * * *$ & & & & & & & & \\
\hline 6. Ignoring asthma & $.16^{* * * *}$ & $.25^{* * *}$ & $.51 * * *$ & $.30 * * *$ & $.17 * * *$ & & & & & & & \\
\hline 7. Worrying asthma & $.31 * * *$ & $.50 * * *$ & $.27 * * *$ & $.33 * * *$ & $.56^{* * * *}$ & $.30 * * *$ & & & & & & \\
\hline \multicolumn{13}{|l|}{ Quality of Life } \\
\hline 8. Medication & $-.34 * * *$ & $-.36^{* * *}$ & $-.22 * * *$ & $-.18 * * *$ & $-.33 * * *$ & $-.25^{* * *}$ & $-.42 * * *$ & & & & & \\
\hline 9. Physical activities & $-.49 * * *$ & $-.47 * * *$ & $-.13 * *$ & $-.13 * *$ & $-.31 * * *$ & $-.14 * *$ & $-.40 * * *$ & $.53 * * *$ & & & & \\
\hline 10. Emotions & $-.44 * * *$ & $-.49 * * *$ & $-.18 * * *$ & $-.20 * * *$ & $-.35 * * *$ & $-.20 * * *$ & $-.53 * * *$ & $.63 * * *$ & $.75^{* * *}$ & & & \\
\hline 11. Social interaction & $-.40 * * *$ & $-.47 * * *$ & $-.16 * * *$ & $-.25 * * *$ & $-.37 * * *$ & $-.16 * * *$ & $-.50 * * *$ & $.59 * * *$ & $.60 * * *$ & $.72 * * *$ & & \\
\hline 12. Positive effects & $.13^{* *}$ & $.26^{* * *}$ & $-.15 * * *$ & $.34 * * *$ & $.31 * * *$ & .06 & $.21 * * *$ & $-.20 * * *$ & $-.27 * * *$ & $-.36 * * *$ & $-.43 * * *$ & \\
\hline Mean & 1.77 & 1.80 & 1.91 & 2.19 & 1.72 & 1.87 & 1.64 & 5.41 & 4.62 & 5.31 & 5.51 & 3.54 \\
\hline SD & 0.38 & 0.68 & 0.69 & 0.70 & 0.66 & 0.75 & 0.67 & 1.18 & 1.43 & 1.43 & 1.16 & 1.39 \\
\hline
\end{tabular}

Note: $* * P<0.01 ; * * * P<0.001$ (2-tailed)

Table 3 Standardised factor loadings of latent factors overall $Q O L$ and Severity and interrelations of coping strategies and QOL

Factor Loadings:

Severity $\rightarrow$ parcel 1

Severity $\rightarrow$ parcel 2

.60

Severity $\rightarrow$ parcel 3

.72

Overall QOL $\rightarrow$ QOL medication

Overall QOL $\rightarrow$ QOL physical activities $\quad .80$

Overall QOL $\rightarrow$ QOL emotions $\quad .91$

Overall QOL $\rightarrow$ QOL social interaction $\quad .80$

Interrelations

Coping Restricted lifestyle $\leftrightarrow$ Coping Hiding asthma

Coping Restricted lifestyle $\leftrightarrow$ Coping Positive reappraisal

Coping Restricted lifestyle $\leftrightarrow$ Coping Information seeking

Coping Restricted lifestyle $\leftrightarrow$ Coping Ignoring asthma

Coping Restricted lifestyle $\leftrightarrow$ Coping Worrying about asthma

Coping Hiding asthma $\leftrightarrow$ Coping Positive reappraisal

Coping Hiding asthma $\leftrightarrow$ Coping Information seeking

Coping Hiding asthma $\leftrightarrow$ Coping Ignoring asthma

Coping Hiding asthma $\leftrightarrow$ Coping Worrying about asthma

Coping Positive reappraisal $\leftrightarrow$ Coping Information seeking

Coping Positive reappraisal $\leftrightarrow$ Coping Ignoring asthma

Coping Positive reappraisal $\leftrightarrow$ Coping Worrying about asthma

Coping Information seeking $\leftrightarrow$ Coping Ignoring asthma

Coping Information seeking $\leftrightarrow$ Coping Worrying about asthma

Coping Ignoring asthma $\leftrightarrow$ Coping Worrying about asthma

Overall QOL $\leftrightarrow$ QOL positive effects

$\begin{array}{rl}.12 & <0.01 \\ .34 & <0.001 \\ .36 & <0.001 \\ .20 & <0.001 \\ .42 & <0.001 \\ -.04 & \mathrm{~ns} \\ .00 & \mathrm{~ns} \\ .50 & <0.001 \\ .24 & <0.001 \\ .52 & <0.001 \\ .28 & <0.001 \\ .30 & <0.001 \\ .13 & <0.01 \\ .52 & <0.001 \\ .26 & <0.001 \\ -.35 & <0.001 \\ & \end{array}$

adolescents led a restricted life and the more they worried, the lower their QOL. The positive QOL domain was mainly associated with the coping strategies hiding asthma and positive reappraisal; positive QOL was higher for adolescents who tended to hide their asthma less, and for adolescents who reappraised their disease more positively. In contrast with the finding that restricted lifestyle was related to poorer overall QOL, this coping strategy was associated with better positive effects QOL. Additionally, scores on the positive QOL domain were higher for adolescents with increased information seeking.

\section{The indirect effect of severity on QOL via coping}

Severity was related to all coping strategies; the more severe the asthma, the more the adolescents used the six coping strategies. Severity was directly-and 


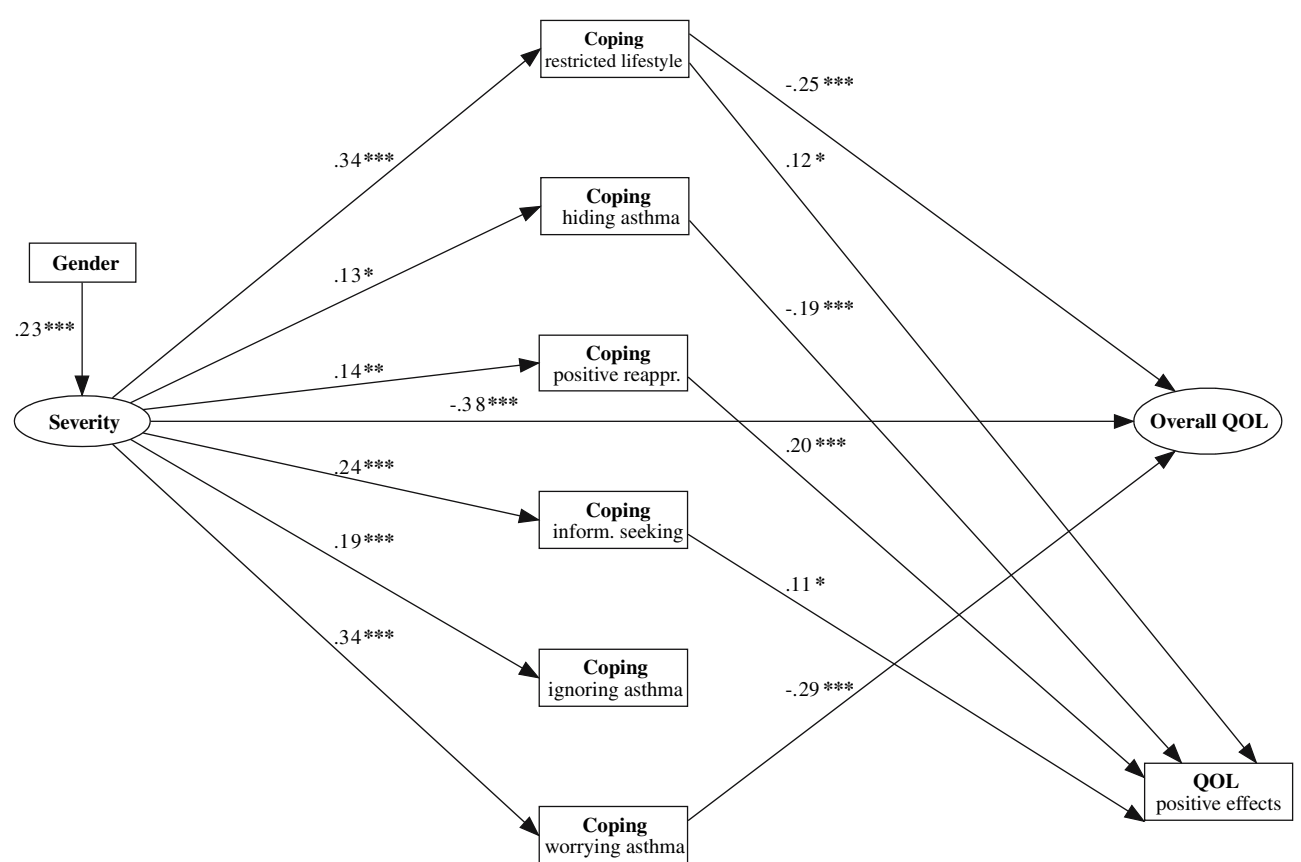

Fig. 2 Structural Equation model with standardised coefficients for the relations between severity, coping and QOL. Only significant paths are given in this figure. Number of distinct sample moments 170; number of distinct parameters to be estimated 104. $N=553, \chi^{2}[66]=174.89, P=0.000, \chi^{2} / \mathrm{df}=2.65$, $\mathrm{CFI}=0.963, \quad \mathrm{RMSEA}=0.055 ; \quad \mathrm{R}^{2}$ overall $\mathrm{QOL}=0.56 ; \mathrm{R}^{2}$ positive QOL $=0.19$. $* P<0.05 ; * * P<0.01 ; * * * P<0.001$ negatively—related to overall QOL. Severity was also indirectly related to overall QOL via the coping strategies restricted lifestyle and worrying about asth$m a$. This indirect path was negative as well: higher severity was related to increased use of these previously mentioned coping styles, which were negatively related to overall QOL. Additionally, the model showed no significant direct path from severity to positive QOL; there were only indirect paths via restricted lifestyle, hiding asthma, positive reappraisal and information seeking. Three of these indirect paths were positive: higher severity scores were positively related to the use of the coping strategies restricted lifestyle, positive reappraisal and information seeking, which in turn had a positive relation with positive QOL. However, one of the indirect paths was of a negative nature: higher severity was related with increased hiding asthma, which in turn had a negative relation with positive QOL.

\section{Additional analyses}

To exclude the possibility that the proportion of variance explained by the model is caused by the association between symptom severity and QOL, an additional model was tested without severity. This model still fitted the data well, and explained $45 \%$ of the variance of overall QOL and $18 \%$ of positive QOL.

To test whether the strengths of the relationships in the model differed between girls and boys, multigroup analyses with chi-square difference testing was applied. No significant differences were found in the factor loadings or regression weights, indicating that the strengths of the paths in the model did not differ between boys and girls.

\section{Discussion}

This study has found that asthma symptom severity is negatively associated with asthma-specific QOL in adolescents with asthma. The findings provide support for the hypothesis that coping mediates the negative relationship between severity and QOL. Thus, the use of adequate coping strategies to deal with symptoms of asthma appears to prevent adolescents with asthma from experiencing a decline in their QOL.

How do adolescents with asthma cope with their asthma? The most reported coping strategy was positive reappraisal, indicating that adolescents with asthma try to actively cope with their asthma. However, the two next most frequently used strategies are passive coping strategies: hiding asthma and ignoring asthma, 
which shows that adolescents also commonly use avoidant coping strategies. Worrying about asthma was the least used coping strategy. Similar results have been found in a study on coping in chronically ill adolescents, where avoidance and confrontation were the most frequently used coping styles, and depressive reactions the least used [31]. Although there is similar ordering of coping styles between boys and girls, this study also found gender differences in coping. Girls reported using all coping strategies more often than boys, consistent with a study by Wilson et al. [32] which showed that female adolescents reported to use all studied coping styles (avoidant coping, problem-focused coping, and emotional coping) more often than males.

While symptom severity was only indirectly related to positive effects QOL, symptom severity was both directly and indirectly related to overall QOL. When adolescents experience more symptoms, they have poorer overall QOL. This association has been shown in previous studies, both among adults [6] and adolescents $[5,7,8]$. Due to the cross-sectional nature of this study, we cannot determine the direction of the associations found. Part of this association may be explained by good QOL leading to better selfmanagement of asthma, thereby reducing symptoms of asthma.

Although we have to be careful with interpreting the directionality of the identified relationships, these results suggest that coping mediates the effect of symptom severity on QOL, indicating that interventions based on changing coping strategies may be an approach to improve QOL in adolescents with asthma. A review on the impact of interventions designed to improve coping by de Ridder and Schreurs [33] suggests such approaches offer 'a promising framework for the development of psychosocial care for chronically ill patients'.

Leading a restricted lifestyle was one of the coping strategies found to mediate the relation between severity and overall $Q O L$. When adolescents experience more symptoms, they avoid situations and triggers, which in turn is associated with poorer overall $Q O L$. This restricted lifestyle may cause adolescents to avoid peer activities, such as parties or extracurricular activities, and thereby decrease their satisfaction with life. Similar results are found in adults with asthma, where avoidant coping was shown to be related to lowered QOL $[9,10]$.

Another mediating coping strategy was worrying about asthma. Increased symptom severity was related to more worrying, which in turn decreased overall QOL. This is consistent with the study of Hesselink et al. [10], who showed a negative association between emotional coping and QOL among adults with asthma.

To improve QOL in adolescents with asthma, interventions could be aimed at decreasing a restricted lifestyle and worrying about asthma. This might be done by teaching adolescents to recognize their triggers of asthma and by promoting greater self-management of their illness. This could increase their selfefficacy expectations, such that by feeling more in control of asthma, adolescents may feel less inclined to avoid particular situations such as parties and playing sports. This increased self-efficacy might also reduce worrying about asthma. In addition, interventions could also focus on cognitive restructuring and relaxation to reduce worrying, and thereby enhance QOL among adolescents with asthma.

With regard to the positive $Q O L$ domain, hiding asthma and positive reappraisal were the two mediating coping strategies most strongly related to positive $Q O L$. Adolescents with worse asthma severity tended to hide their asthma more, which was related to lower positive $Q O L$. This may result in fewer people from their social environment being aware of their condition, which may reduce the opportunities for social support, and potentially thus experience fewer positive effects. Interventions that target individuals, such as social skills training or assertiveness training, or interventions that target schools, such as the Asthma Friendly Schools Program [34], might help adolescents feel more able to inform others about their illness. Peer support groups like ChIPS (Chronic Illness Peer Support) [35] and the Triple A program [36] may also help to build resilience and enhance self-confidence and self-acceptance, making it easier for adolescents to be open rather than hide their asthma.

Positive reappraisal was the coping strategy most strongly related to positive QOL. Adolescents who tried to see positive aspects of their asthma also perceived more positive effects. Part of this association may be due to overlap in constructs. However, studies showed that optimistic persons have greater friendship networks [37], longer friendships [38], and perceive more social support [37]. Therefore we assume that part of the association found in this study is not due to overlap but due to a more positive mindset of the adolescents, making it easier for their social environment to react in a positive way to the adolescent. This is in line with Folkman \& Moskowitz [39], who emphasize the role of positive reappraisal in coping with chronic illness. Although positive reappraisal seems to affect positive $Q O L$ among adolescents with asthma, this coping strategy might be hard to target in interventions because of the effect of personality 
factors such as optimism, which have been shown to be related to emphasizing positive aspects of stressful situations [40].

This is the first study to describe the relation between coping strategies and QOL in adolescents with asthma. A strength of this study is the use of a large community sample, which is ruling out the risk of a self-selection bias, and includes adolescents with all levels of asthma severity. This study also used validated instruments specifically designed for adolescents with asthma. The use of sophisticated statistical methods to test relations between severity, coping and QOL allowed testing of both direct and indirect paths, testing group differences in these direct and indirect paths, and decreasing measurement error by using latent factors.

Some of the standardized regression coefficients in the model are weak but significant, which may be partly due to the larger sample size. However, the strengths of these paths are similar to those found in other studies on the relation between coping and QOL [10], and the total model explains $56 \%$ of the variance in overall QOL and 19\% in positive QOL. When severity was excluded from the model, the model still explained $45 \%$ of the variance of overall QOL and $18 \%$ of positive QOL, indicating the clinical relevance of coping strategies in QOL among adolescents with asthma. Another limitation of this study is the low reliability of three of the coping subscales, which could have influenced the model. However, coping is a difficult construct to measure, and other coping scales like COPE [41], which are frequently used [37] show Cronbachs alpha's in the same range as those reported in the present study. Furthermore, the alpha's reported in the present study are in line with the validation study ( $\alpha$ ranging from .63 to .84) [23]. A third limitation is that due to the cross-sectional nature of this study, we cannot determine the direction of the associations found. For instance, we assume that severity influences coping, but we cannot rule out the possibility that coping affects symptom severity as well. Thus hiding asthma could lead to a decrease in adherence due to not taking medication in peer settings, which in turn could lead to an increase in symptoms. In regard to the relations between QOL and coping, while worrying about asthma may lead to poorer overall QOL, it is also possible that lower QOL may result in adolescents worrying more about their asthma. Longitudinal designs could provide more insight into the underlying mechanism of the relations found in this study. Further research would also benefit from including personality factors, such as optimism, and other measures of psychosocial well being, such as depression, which may affect both QOL and coping strategies. Finally, since the responsibility for the management of asthma is transferred from parent to adolescent, further studies that include family factor could also provide fresh insights.

Acknowledgements This research was funded by a grant from the Dutch Asthma Foundation. The contribution of Rutger Engels was supported by the Netherlands Organisation for Scientific Research. The authors are grateful to all adolescents who participated in the project.

\section{References}

1. Lenney, W. (1997). The burden of pediatric asthma. Pediatric Pulmonology Supplement, 15, 13-16.

2. Price, J. F. (1996). Issues in adolescent asthma: What are the needs? Thorax, 51 (Suppl 1), S13-17.

3. Graetz, B., \& Shute, R. (1995). Assessment of peer relationships in children with asthma. Journal of Pediatric Psychology, 20, 205-216.

4. Schipper, H., Clinch, J., \& Powell, V. (1990). Definitions and conceptual issues. In B. Spilker (Ed.), Quality of life assessment in clinical trials. New York: Raven Press, pp. 11-24.

5. Sawyer, M. G., Spurrier, N., Whaites, L., Kennedy, D., Martin, A. J., \& Baghurst, P. (2001). The 2 relationship between asthma severity, family functioning and the healthrelated quality of life of children with asthma. Quality of Life Research, 9, 1105-1115.

6. Horak, E., Sawyer, S. M., Roberts, M., et al. (2005). Impact of disease severity on quality of life in adults with asthma. Wiener Klinische Wochenschrift, 117, 462-467.

7. Warschburger, P., Busch, S., Bauer, C. P., Kiosz, D., Stachow, R., \& Petermann, F. (2004). Health-related quality of life in children and adolescents with asthma: results from the ESTAR Study. Journal of Asthma, 41, 463-470.

8. Somerville, A., Knopfli, B., \& Rutishauser, C. (2004). Health-related quality of life in Swiss adolescents with asthma. Validation of the AAQOL-D and comparison with Australian adolescents. Swiss Medical Weekly, 134, 91-96.

9. Adams, R.J., Wilson, D., Smith, B. J., \& Ruffin, R. E. (2004). Impact of coping and socioeconomic factors on quality of life in adults with asthma. Respirology, 9, 87-95.

10. Hesselink, A. E., Penninx, B. W., Schlosser, M. A., et al. (2004). The role of coping resources and coping style in quality of life of patients with asthma or COPD. Quality of Life Research, 13, 509-518.

11. Lazarus, R. S., \& Folkman, S. (1984). Stress, appraisal, and coping. New York: Springer.

12. Ritsner, M., Ben-Avi, I., Ponizovsky, A., Timinsky, I., Bistrov, E., \& Modai, I. (2003). Quality of life and coping with schizophrenia symptoms. Quality of Life Research, 12, $1-9$.

13. Van De Ven, M. O., Engels, R. C., \& Van Den Eijnden, R. J. (2006) Atopic diseases and related risk factors among Dutch adolescents. European Journal of Public Health, 16, 549-558.

14. Williams, C. (2000). Doing health, doing gender: teenagers, diabetes and asthma. Social Science and Medicine, 50, 387-396.

15. Otten, R., Engels, R. C., \& Van Den Eijnden, R. J. (2005). Parental smoking and smoking behavior in asthmatic and nonasthmatic adolescents. Journal of Asthma, 42, 349-55. 
16. Asher, M. I., Keil, U., Anderson, H. R., et al. (1995). International Study of Asthma and Allergies in Childhood (ISAAC): rationale and methods. The European Respiratory Journal, 8, 483-491.

17. Wieringa, M. H., Weyler, J. J., Van Bever, H. P., Nelen, V. J., \& Vermeire, P. A. (1999). Gender differences in respiratory, nasal and skin symptoms, 6-7 versus 13-14-year-old children. Acta Paediatrica, 88, 147-149.

18. Pearce, N., Weiland, S., Keil, U., et al. (1993). Self-reported prevalence of asthma symptoms in children in Australia, England, Germany and New Zealand: an international comparison using the ISAAC protocol. The European Respiratory Journal, 6, 1455-1461.

19. Shaw, R., Woodman, K., Ayson, M., et al. (1995). Measuring the prevalence of bronchial hyper-responsiveness in children. International Journal of Epidemiology, 24, 597-602.

20. Jenkins, M. A., Clarke, J. R., Carlin, J. B., et al. (1996). Validation of questionnaire and bronchial hyperresponsiveness against respiratory physician assessment in the diagnosis of asthma. International Journal of Epidemiology, 25, 609-16.

21. Redline, S., Gruchalla, R. S., Wolf, R. L., et al. (2004). Development and validation of school-based asthma and allergy screening questionnaires in a 4-city study. Annals of Allergy, Asthma and Immunology, 93, 36-48.

22. Rutishauser, C., Sawyer, S. M., Bond, L., Coffey, C., \& Bowes, G. (2001). Development and validation of the Adolescent Asthma Quality of Life Questionnaire (AAQOL). The European Respiratory Journal, 17, 52-58.

23. Aalto, A. M., Harkapaa, K., Aro, A. R., \& Rissanen, P. (2002). Ways of coping with asthma in everyday life: validation of the Asthma Specific Coping Scale. Journal of Psychosomatic Research, 53, 1061-1069.

24. Maes, S., \& Schlösser, M. (1987). The role of cognition and coping in health behaviour outcomes of asthmatic patients. Current Psychological Research Reviews, 6, 79-90.

25. Arbuckle, J. L., \& Wothke, W. (2003). AMOS 5.0 user's guide. Chicago: SPSS.

26. Likert, R. (1932). A technique for the measurement of attitudes. Archives of Psychology, 140, 1-55.

27. Torgerson, W. S. (1958). Theory and Methods of Scaling. New York: John Wiley \& Sons.

28. MacCallum, R. C., Browne, M. W., \& Sugawara, H. M. (1996). Power analysis and determination of sample size for covariance structure modeling. Psychological Methods, 1, 130-149.

29. Bandalos D., \& Finney S. (2001). Item parceling issues in structural equation modeling. In G. Marcoulides \&
R. Schumacker (Eds.), Advanced structural equation modeling: New developments and techniques. Mahwah, NJ: Lawrence Erlbaum Associates.

30. Kaplan, D. (2000). Structural equation modelling: Foundations and extensions. Newbury Park, CA: Sage.

31. Meijer, S. A., Sinnema, G., Bijstra, J. O., Mellenbergh, G. J., \& Wolters, W. H. G. (2002). Coping styles and locus of control as predictors for psychological adjustment of adolescents with a chronic illness. Social Science and Medicine, $54,1453-1461$.

32. Wilson, G. S., Pritchard, M. E., \& Revalee, B. (2005). Individual differences in adolescent health symptoms: The effects of gender and coping. Journal Adolescence, 28, 369379.

33. De Ridder, D., \& Schreurs, K. (2001). Developing interventions for chronically ill patients: is coping a helpful concept? Clinical Psychology Review, 21, 205-40.

34. Sawyer, S. M. (2006). Asthma friendly schools: the importance of school policy for children with asthma. Journal of Paediatrics and Child Health, 42, 483-485.

35. Olsson, C. A., Boyce, M. F., Toumbourou, J. W., \& Saywer, S. M. (2005). The role of peer support in facilitating pyschosocial adjustment to chronic illness in adolescence. Clinical Child Psychology and Psychiatry, 10, 78-87.

36. Shah, S., Peat, J. K., Mazurski, E. J., et al. (2001). Effect of peer led programme for asthma education in adolescents: cluster randomised controlled trial. British Medical Journal, 322, 583-585.

37. Brissette, I., Scheier, M. F., \& Carver, C. S. (2002). The role of optimism in social network development, coping, and psychological adjustment during a life transition. Journal of Personality and Social Psychology, 82, 102-111.

38. Geers, A. L., Reilley, S. P., \& Dember, W. N. (1998). Optimism, pessimism, and friendship. Current Psychology, 17, 3-19.

39. Folkman, S., \& Moskowitz, J. T. (2000). Positive affect and the other side of coping. The American Psychologist, 55, 647654.

40. Scheier, M. F., Weintraub, J. K., \& Carver, C. S. (1986). Coping with stress, Divergent strategies of optimists and pessimists. Journal of Personality and Social Psychology, 51, 1257-1264.

41. Carver, C. S., Scheier, M. F., \& Weintraub, J. K. (1989). Assessing coping strategies: A theoretically based approach. Journal of Personality and Social Psychology, 56, 267-283. 